\title{
Damping Ring Kickers for the Next Linear Collider ${ }^{*}$ \\ C. Pappas, R. Cassel \\ Stanford Linear Accelerator Center
}

\begin{abstract}
The Next Linear Collider (NLC) uses a damping ring for the electron beam, a pre-damping ring and a main damping ring for the positron beam to reduce the beam emittances. The requirements of the main damping ring kickers are to bend a $2 \mathrm{GeV}$ beam by an angle of $2 \mathrm{mrad}$ over a length of $1.2 \mathrm{~m}$. This results in a required field of 139 G. The magnet aperture is $30 \times 30 \mathrm{~mm}$. The predamping ring kicker requirements are based on a $2 \mathrm{GeV}$ beam with a bend angle of $8 \mathrm{mrad}$ in $1.2 \mathrm{~m}$, or a field of $308 \mathrm{G}$. The magnet aperture is $62 \times 45 \mathrm{~mm}(\mathrm{H} \times \mathrm{V})$. A pulse width is $130 \mathrm{~ns}$ with rise and fall times of less than $60 \mathrm{~ns}$ is the same for both the pre-damping ring and main damping ring kickers. The three rings operate at a $180 \mathrm{~Hz}$ repetition rate ${ }^{1}$.
\end{abstract}

The kicker magnets being developed to meet these requirements consist of two strip line conductors in the vacuum chamber, for the pre-damping ring kickers they may be loaded with ferrite, to give a matching impedance of $25 \Omega$. The buses are separated from magnetic flux linkage by a grounded flux excluder, which also serves as a low impedance return for the beam current. Both busses of the magnet are driven in parallel from the same modulator and are grounded at the end opposite the feed. The modulator uses two IGBT stacks which both act as opening switches in order to meet the rise time requirements.

\section{Damping Ring Kicker Magnets}

Several types of magnets were considered to meet the difficult rise time parameters for the damping ring kickers. An air core, strip line, matched impedance magnet was decided upon because of its simplicity, low cost, and it uses no non-linear materials ${ }^{2}$. The magnet is made from a slotted pipe which is housed in the vacuum chamber. A metal strip is brazed onto the top and bottom of the slotted pipe to prevent magnetic coupling between the two busses during the pulse rise time. A drawing of the magnet is shown in Figure 1. This magnet was analyzed with the electro-magnetic field solver MAXWELL. The impedance of the magnet was calculated to be $32 \Omega$, and the one way transit time to be 3.49 ns per meter. A plot of the $\mathbf{B}$ field magnitude with a drive current of one ampere is shown in Figure 2.

*Work supported by Department of Energy contract DEAC03-76SF515.

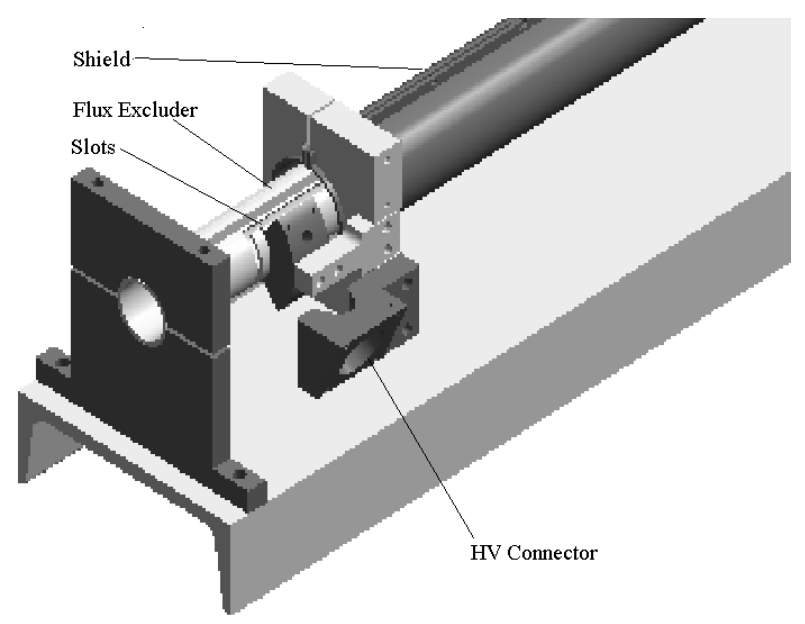

Figure 1. Proto type slotted kicker magnet.

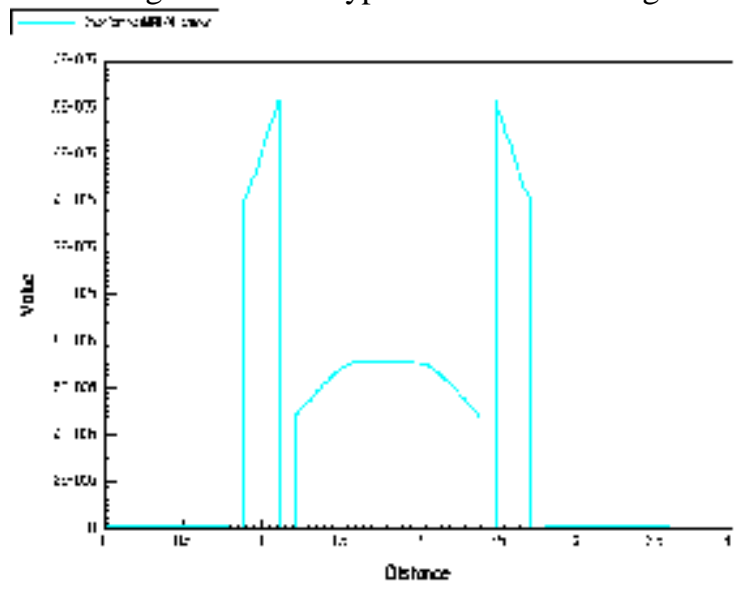

Figure 2. Calculated $\mathbf{B}$ field magnitude.

A one meter long, stainless steel magnet was built and tested. An oscillograph of the magnet current, $\mathbf{B}$ dot, and the $\mathbf{B}$ field are shown in Figure 3. It can be noted from this data that the current in the magnet is constant while the $\mathbf{B}$ field rises as time progresses. After eliminating the B dot probe, electric fields, digitization error, and other measurements errors, it was determined that the slope of the $\mathbf{B}$ field might be explained by skin depth effects caused by the stainless steel. Because the resistivity of stainless is approximately fifty times greater than copper, the steel magnet structure will allow the currents to penetrate further into the conductors, essentially increasing the width of the slits, and hence the magnetic field. The magnetic filed was recalculated allowing the currents to penetrate further into the magnet busses by increasing the permeability of the conductors. It was found that the magnetic field intensity increased by a 
factor of approximately $12 \%$ with a decrease in the permeability of the conductors used in the calculations for 0.001 to 0.01 . Based upon these calculations the magnet was plated with 10 mils of copper, and tested again. The results of this testing are shown in Figure 4, where it is clearly seen that the $\mathbf{B}$ field no longer increases during the pulse.

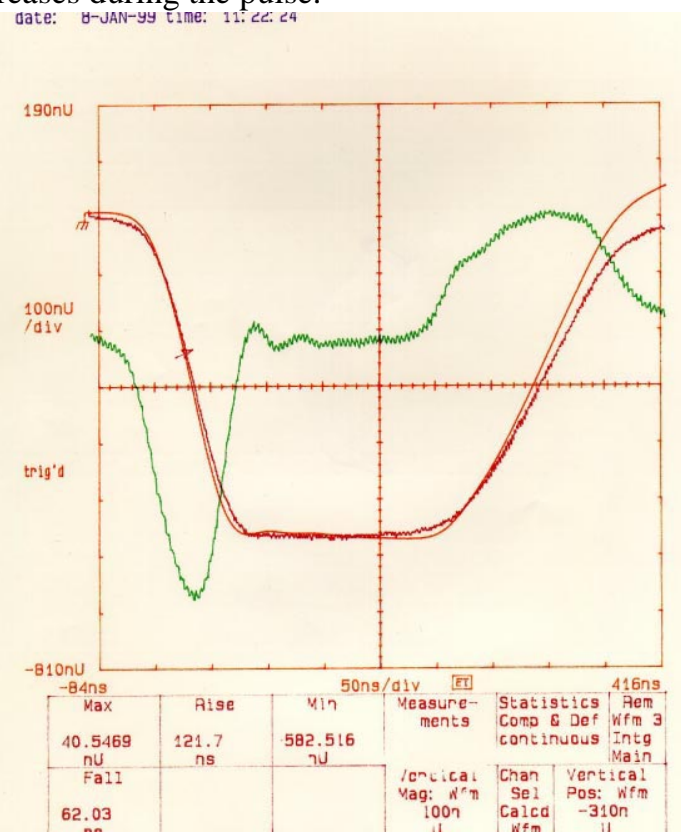

Figure 3. $\mathbf{B}$ field and current in the stainless steel magnet.

Further measurements were then made both with and without the shield in place, and the results where compared with the calculated values of the magnetic gain. The B dot probe was rotated $180{ }^{\circ}$ for each measurement and the traces were subtracted to eliminate the effects of electric field. Figure 5 shows the magnet current, and integrated $\mathbf{B} \cdot 1$ curve with the shield, and Figure 6 shows the same curves without the shield. The measured gain of the magnet with the shield is approximately $69 \mathrm{mG} / \mathrm{A}$, while the calculated gain is approximately $71 \mathrm{mG} / \mathrm{A}$. With the shield removed the gain is measured to be approximately $118 \mathrm{mG} / \mathrm{A}$, and the computed gain is 117 $\mathrm{mG} / \mathrm{A}$.

The impedance of the magnet was then measured with a TDR and compared with the calculated values. The measured impedance with the shield was approximately $35 \Omega$ and the computed impedance was $32 \Omega$. Without the shield we measured the impedance to be $61 \Omega$, and calculated it to be greater than $53 \Omega$. Initial beam impedance measurements performed at Lawrence Berkeley National Laboratory indicate that the magnet structure will not seriously impact beam performance.

\section{The IGBT Based Modulator}

A solid state modulator which uses a set of IGBTs as the switching element is being developed. A schematic of the

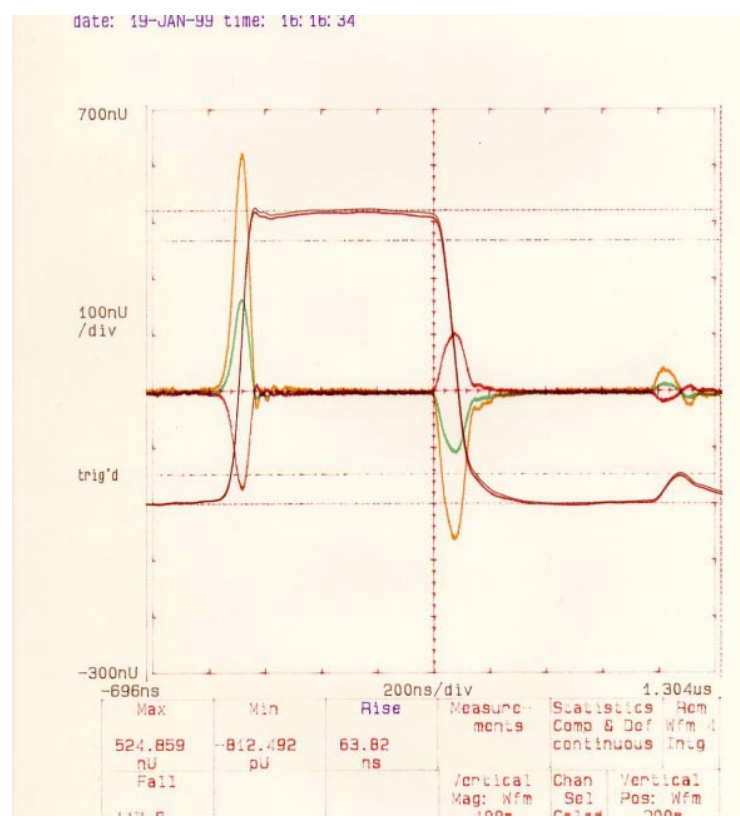

Figure 4. B field and current of the copper plated magnet.

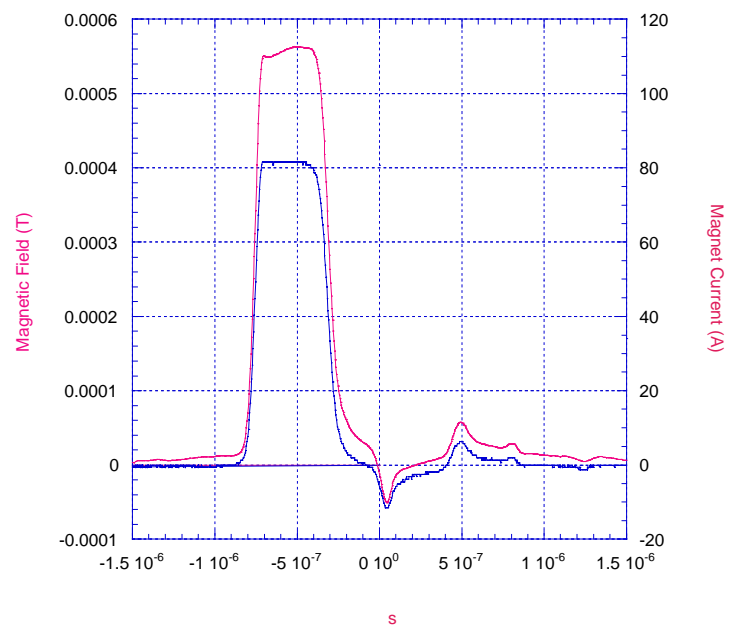

Figure 5. Magnet current and integrated field with shield.

circuit is shown in Figure 7. The operation of the circuit is as follows. The IGBT stack to the right is turned on to begin charging of the capacitors through the diodes. When the capacitors are fully charged the IGBT stack to the left is turned on, putting the full voltage across the saturable reactor. Just as the core of the reactor saturates, the IGBTs to the right are opened, transferring the current to the load. The pulse is ended by opening the IGBT stack on the left. This switching scheme is done to utilize the opening characteristics of the IGBT modules, which are faster than the closing speed. A model of the circuit has been built which uses three Eupec BSM-300GA170DN2S, $1700 \mathrm{~V}$, IGBTs in each stack. The circuit has been fully tested, and was used as the pulser for the data in this paper $^{3}$. Higher voltage devices $(3300 \mathrm{~V})$ have also been purchased, and have been found to have better 
switching characteristics. We are currently waiting delivery of $6500 \mathrm{~V}$ devices ${ }^{4}$.

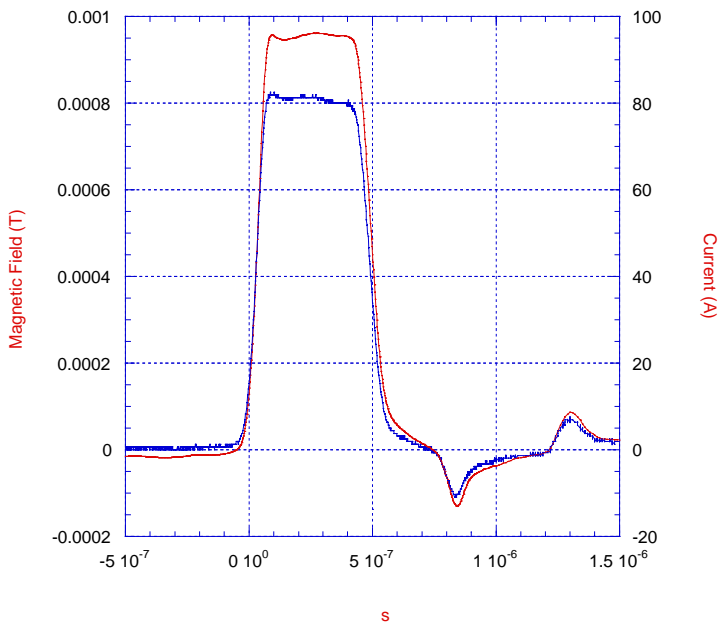

Figure 6. Magnet current and integrated field without shield.

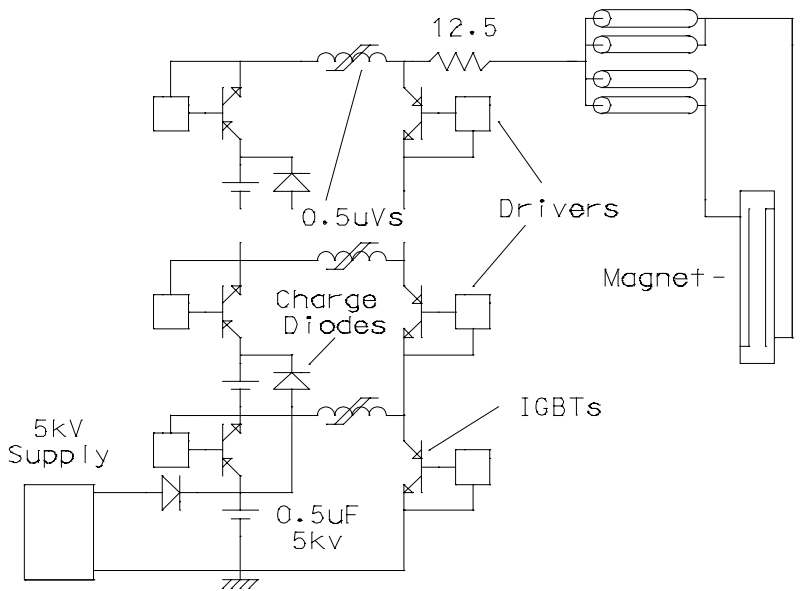

Figure 7. Schematic of solid state modulator.

\section{The Pre-damping Ring Kickers.}

Work has begun on the pre-damping ring kickers, with several different types of magnets under consideration. A slotted pipe kicker similar to the one above, a similar magnet which would be ferrite loaded, and a traveling wave C-magnet similar to the SLAC damping ring kickers $^{5}$ are being studied. With the simple geometry shown in Figure 8, the magnetic gain and impedance of both an air core, and ferrite loaded kicker magnet have been calculated. For the air core magnet the gain is approximately $35 \mathrm{mG} / \mathrm{A}$, with an impedance of $46 \Omega$. This magnet would therefore require the IGBT to switch 4400 A, if a circuit similar to Figure 7 were used. When the magnet is loaded with ferrite, the magnetic gain is increased to approximately $160 \mathrm{mG} / \mathrm{A}$, and the impedance is reduced to $27 \Omega$, therefore the IGBT current would be reduced to $963 \mathrm{~A}$. The field is also slightly more uniform if the magnet is load with ferrite. One problem with a ferrite loaded magnet, besides complexity and cost, is that some method would have to be incorporated to make sure that there are no large air gaps between the ferrite and the conductors. The ferrite could be put into the vacuum chamber and sandwiched between the conductors and a sheet of soft metal such as indium, or the ferrite could be potted between the busses, which would necessitate a ceramic beam pipe. Another problem is that he ferrite would load the beam, which would require cooling for the ferrite.

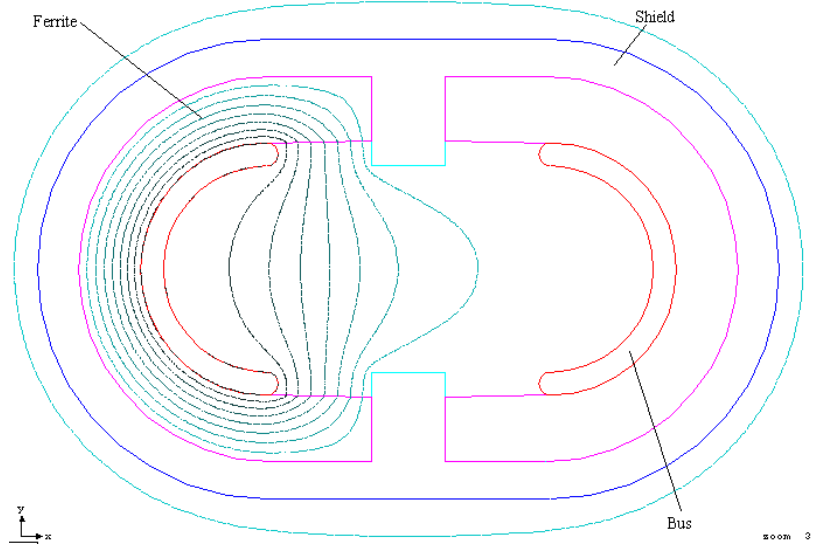

Figure 8. Magnetic flux lines in ferrite loaded kicker.

\section{Conclusions}

A slotted pipe kicker magnet is being developed at SLAC for the NLC damping rings, which does not appear to have any major problems. It can be driven by a solid state, IGBT based modulator. The overall cost and reliability of such a kicker system should be much better than for a conventional type of kicker system. Development of a pre-damping ring kicker system has begun, with several options available. Further research is required before a decision can be reached as to which technology is preferable.

\section{References}

[1] The Next Linear Collider Damping Ring Complex, M. Ross, etal, this conference.

[2] A Slotted-pipe Kicker for High-current Storage Rings, G. Blokesch, etal, Nuclear Instruments and Methods in Physics Research A 338 (1994).

[3] A Solid State Induction Modulator for SLAC SLC, R. Cassel, etal, this conference.

[4] Eupec Electronic Data Book, 1999, Eupec Inc., Lebanon, NJ.

[5] Kicker Systems for the Stanford Linear Collider, T. Mattison, etal, 1990 Journal, Particle Accelerators. 